\title{
Solitons of the KP equation in dusty plasma with variable dust charge and two temperature ions : energy and stability
}

\author{
Hamid Reza Pakzad* and Kurosh Javidan ${ }^{1}$ \\ Department of Physics, Azad University of Bojnourd, Bojnourd, Iran \\ ${ }^{1}$ Department of Physics, Ferdowsi University of Mashhad, 91775-1436 Mashhad, Iran \\ E-mail : pakzad@bojnourdiau.ac.ir
}

Received 13 May 2008, accepted 29 September 2008

\begin{abstract}
The propagation of nonlinear waves in dusty plasmas with variable dust charge and two temperature ions is analyzed. By using the reductive perturbation theory, the Kadomtsev-Petviashivili (KP) equation is derived. A Sagdeev potential has been investigated. This potential is used to study the stability conditions for existence of solitonic solutions. Also, it is shown that a rarefactive soliton can exist in most of the cases. The energy of the soliton has been calculated and by using the standard normal-mode analysis a linear dispersion relation has been obtained. The effects of variable dust charge on the amplitude, width and energy of soliton and its effects on the angular frequency of linear wave are also discussed.
\end{abstract}

Keywords : dust, soliton, KP

PACS Nos. : $52.35 \mathrm{Bj} ; 52.35 \mathrm{Mw} ; 52.35 . \mathrm{S}$

\section{Introduction}

Solitary waves and solitons represent one of the interesting and famous aspects of nonlinear phenomena in spatially extended systems. They appear as specific types of localized solutions of various nonlinear partial differential equations and possess several important properties.

Dusty plasmas are an ideal medium for creating solitary waves and solitons. Such environments have been observed in the earth's magnetosphere, cometary tail, planetary rings and so on [1-3]. Moreover study of dusty plasma media is very attractive because of their theoretical features and also their applications.

* Corresponding Author 
The low frequency oscillations in dusty plasmas have been studied in references $[4,5]$. The effect of dust temperature has been investigated in reference [6] and the normal modes of plasmas because of the existence of heavy dust particles have been modified in reference [7]. In most investigations reductive perturbation method has been used for deriving the 'kdv' or 'mkdv' equation in one-dimensional case [8-10] and also for KP equation in higher dimensions [11]. The charging process of dust particles is an important effect which has been investigated in $[8,9]$. This phenomenon was also studied by using semiinverse method, applied to ion-acoustic plasma waves in [10].

In the present paper, the dusty plasma with the variable dust charge and two temperature ions has been considered. By using the reductive perturbation method (RPM) on two dimensional unmagnetized case of this system, one can obtain the KP equation. Balancing between nonlinear and dispersion effects can result in the formation of symmetrically solitary waves. The KP equation has been obtained for dust acoustic waves in hot dusty plasmas and also in dust ion acoustic dusty plasmas [12, 13]. In section 2, the basic set of equations is introduced and in section 3, the KP equation has been derived. Section 4 contains discussion on soliton solution and its stability conditions. In section 5 we obtain energy of the soliton. The linear dispersion relation and effects of variable dust charge on this relation have been discussed in this section. Conclusions and remarks are given in section 6 .

\section{Basic equations}

We consider the propagation of dust acoustic waves in collisionless, unmagnetized dusty plasma consisting of electrons, two temperature ions and high negatively charged dust grains. Total charge neutrality at equilibrium requires that

$$
n_{0 e}+n_{0 d} Z_{0 d}=n_{0 i l}+n_{0 i h}
$$

where $n_{0 \mathrm{e}}, n_{0 d^{\prime}}, n_{\text {oil }}$ and $n_{\text {oih }}$ are the equilibrium values of electrons, dust, lower temperature ions and higher temperature ions number densities respectively. $Z_{0 d}$ is the unperturbed number of charges on the dust particles. The following set of normalized two dimensional equations of continuity, motion for the dust and Poisson, describe dynamics of dust acoustic wave

$$
\begin{aligned}
& \frac{\partial u_{d}}{\partial t}+u_{d} \frac{\partial u_{d}}{\partial x}+v_{d} \frac{\partial u_{d}}{\partial y}=Z_{d} \frac{\partial \phi}{\partial x} \\
& \frac{\partial v_{d}}{\partial t}+u_{d} \frac{\partial v_{d}}{\partial x}+v_{d} \frac{\partial v_{d}}{\partial y}=Z_{d} \frac{\partial \phi}{\partial y}
\end{aligned}
$$




$$
\frac{\partial^{2} \phi}{\partial x^{2}}+\frac{\partial^{2} \phi}{\partial y^{2}}=Z_{d} n_{d}+n_{e}-n_{d}-n_{i h}
$$

where $u_{d}$ and $v_{d}$ are velocity components of the dust particles in $\mathrm{x}$ and y-directions. $n_{d}, \phi$ and $Z_{d}$ are dust number density, electrostatic potential and variable charge number of dust grains, respectively. Note that all of the above variables have been normalized by $n_{\text {od }}$. $T_{\text {eff }}$ is effective temperature and it is given by :

Also dust acoustic speed, Debye length and inverse of dust plasma frequency are defined by $C_{d}=\left(\frac{Z_{0 d} T_{\text {eff }}}{m_{d}}\right)^{1 / 2}, \lambda_{d}=\left(\frac{T_{\text {eff }}}{4 \pi Z_{0 d}^{2} n_{0 d} e^{2}}\right)^{1 / 2}$ and $\omega_{p d}^{-1}=\left(\frac{m_{d}}{4 \pi n_{0 d} Z_{0 d} e^{2}}\right)^{1 / 2}$ respectively.

Electrons and ions are assumed to be distributed with Maxwell-Boltzmann distribution functions. So related dimensionless number densities for electrons $\left(n_{\mathrm{e}}\right)$, low temperature ions $\left(n_{i l}\right)$ and high temperature ions $\left(n_{i h}\right)$ are :

$\frac{1}{T_{\text {eff }}}=\frac{Z_{0 d} n_{0 d}}{\left(\frac{n_{0 e}}{T_{e}}+\frac{n_{0 i l}}{T_{i l}}+\frac{n_{0 i h}}{T_{i h}}\right)}$

$$
\begin{aligned}
& n_{e}=\frac{n_{0 e}}{n_{0 d} z_{d}} \exp \left(\beta_{1} s \phi\right) \\
& n_{i l}=\frac{n_{0 i l}}{n_{0 d} Z_{d}} \exp (-s \phi) \\
& n_{i h}=\frac{n_{0 i h}}{n_{0 d} Z_{d}} \exp (-\beta s \phi)
\end{aligned}
$$

where

$$
\beta_{1}=\frac{T_{\text {il }}}{T_{e}}, \beta_{2}=\frac{T_{\text {ih }}}{T_{e}}, \quad \beta=\frac{\beta_{1}}{\beta_{2}}=\frac{T_{\text {il }}}{T_{\text {ih }}}, \quad s=\frac{T_{\text {eff }}}{T_{\text {il }}}, \quad \delta_{1}=\frac{n_{\text {oil }}}{n_{0 e}}, \delta_{2}=\frac{n_{\text {oih }}}{n_{0 e}} .
$$

And from (1) it follows

$$
\delta_{1}+\delta_{2}-1 \geq 0 .
$$

The dust charge variable $Q_{d}$ is obtained from the charge-current balance equation [14]

$$
\left(\frac{\partial}{\partial t}+\boldsymbol{V} \cdot \nabla\right) Q_{d}=I_{e}+I_{i l}+I_{i h}
$$


where $\boldsymbol{V}=\left(u_{d}, v_{d}\right)$ and $l_{e}, l_{i l}$ and $l_{i h}$ are the electron and ions (low and high temperature) currents. We further suppose that the streaming velocities of electrons and ions are much smaller than the thermal velocities. Thus and charge-current balance equation (7) reads $I_{e}+I_{i l}+I_{i h} \approx 0$. The electron and ions currents are [15]

$$
\begin{aligned}
& I_{e}=-e \pi r^{2}\left(\frac{8 T_{e}}{\pi m_{e}}\right)^{\frac{1}{2}} n_{e} \exp \left(\frac{e \Phi}{T_{e}}\right) \\
& I_{i l}=e \pi r^{2}\left(\frac{8 T_{i l}}{\pi m_{i}}\right)^{\frac{1}{2}} n_{i l}\left(1-\frac{e \Phi}{T_{i l}}\right) \\
& I_{i h}=e \pi r^{2}\left(\frac{8 T_{i h}}{\pi m_{i}}\right)^{\frac{1}{2}} n_{i h}\left(1-\frac{e \Phi}{T_{i h}}\right)
\end{aligned}
$$

where $\Phi$ denotes the dust grain surface potential relative to the plasma potential [16].

The normalized dust charge, $Z_{d}$ is obtained from

where $\quad \gamma_{1}=\left.\frac{1}{\psi_{0}} \frac{d \psi(\phi)}{d \phi}\right|_{\phi=0}$ and $\gamma_{2}=\left.\frac{1}{\psi_{0}} \frac{d^{2} \psi(\phi)}{d \phi^{2}}\right|_{\phi=0}$.

\section{The derivation of KP equation}

According to the general method of reductive perturbation theory, we choose the independent variables as

$$
\xi=\varepsilon\left(x-v_{0} t\right), \quad \tau=\varepsilon^{3} t, \quad \eta=\varepsilon^{2} y
$$


where $\varepsilon$ is a small dimensionless expansion parameter which characterizes the strength of nonlinearity in the system and $v_{0}$ is the phase velocity of the wave along the $\mathrm{x}$ direction. We can expand physical quantities which have been appeared in (2)-(5), in term of the expansion parameter as

$$
\begin{aligned}
& u_{d}=\varepsilon^{2} u_{1 d}+\varepsilon^{4} n_{2 d}+\ldots . . \\
& v_{d}=\varepsilon^{3} v_{1 d}+\varepsilon^{5} n_{2 d}+\ldots . \\
& \phi=\varepsilon^{2} \phi_{1}+\varepsilon^{4} \phi_{2}+\ldots \\
& Z_{d}=1+\varepsilon^{2} Z_{1 d}+\varepsilon^{4} Z_{2 d} .
\end{aligned}
$$

Also with using (10) one can find

$$
s=\frac{T_{\text {eff }}}{T_{\text {il }}}=\frac{\left(\delta_{1}+\delta_{2}-1\right)}{\delta_{1}+\delta_{2} \beta+\beta_{1}}
$$

substituting (17)-(22) into equations (2)-(5) and collecting terms with same powers of ,

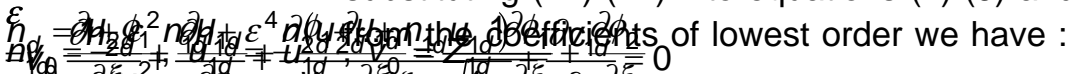

$$
\frac{\partial v_{1 d}}{\partial \xi}=-\frac{1}{v_{0}} \frac{\partial \phi_{1}}{\partial \eta}
$$

And for the higher orders of $\varepsilon$

$$
\frac{\partial^{2} \phi_{1}}{\partial \xi^{2}}=Z_{2 d}+Z_{1 d} n_{1 d}+n_{2 d}+\phi_{2}-\frac{1}{2}\left(\delta_{1}+\delta_{2} \beta^{2}-\beta_{1}^{2}\right) \frac{s^{2}}{\left(\delta_{1}+\delta_{2}-1\right)} \phi_{1}^{2}
$$


The KP equation is derived from the above equations

$$
\frac{\partial}{\partial \xi}\left[\frac{\partial \phi_{1}}{\partial \tau}+a \phi_{1} \frac{\partial \phi_{1}}{\partial \xi}+b \frac{\partial^{3} \phi_{1}}{\partial \xi^{3}}\right]+c \frac{\partial^{2} \phi_{1}}{\partial \eta^{2}}=0
$$

where $a=\frac{v_{0}^{3}}{2}\left[\left(\delta_{1}+\delta_{2} \beta^{2}-\beta_{1}^{2}\right) \frac{\left(\delta_{1}+\delta_{2}-1\right)}{\left(\delta_{1}+\delta_{2} \beta+\beta_{1}\right)^{2}}-2 \gamma_{2}\right]+\frac{3}{2} \gamma_{1} v_{0}-\frac{3}{2 v_{0}}, b=\frac{v_{0}^{3}}{2}, c=\frac{c_{0}}{2}$.

From (10) one can find that $\beta_{1}, \beta<1$. Notice that the derived parameter "a" is different from what has been reported in [11]. Our calculation shows that what has been appeared in [11] can not be correct.

Let us examine sign of "a" which has been defined in (17). Parameter "a" reaches its maximum where the first term becomes maximum and the second term attains its minimum value. The first term is maximum when_. Thus for and "a" is maximal. We choose and in this case "a" is

$$
a=\frac{1}{2}\left[\left(\delta_{1}+\delta_{2} \beta^{2}-\beta_{1}^{2}\right) \frac{\left(\delta_{1}+\delta_{2}-1\right)}{\left(\delta_{1}+\delta_{2} \beta+\beta_{1}\right)^{2}}-3\right]
$$

Obviously $\left(\delta_{1}+\delta_{2} \beta^{2}-\beta_{1}^{2}\right)$ is always less than , but for term we have

It is clear that above term is less than 1 if $\delta_{1}<\left(\left(1+\beta_{1}\right) /(1-\beta)\right)$ and in this case "a" is always negative and rarefactive solitons always exist. Also above mentioned term is more than 1 if and in this case "a" can get positive or negative values and in thse cases both compressive and rarefactive solitary waves can be propagated. Figure (1-4) show the variation of "a" with respect fo different values of and $\delta_{2}$.

In Figure 1 "a" is plotted as a function of $\beta$ and when $\delta_{1}=1, \delta_{2}=4$ and $v_{0}=1$.

Figure 2 presents "a" as a function of and when $\delta_{1}=11, \beta_{2}=0.01$ and Figure 3 shows "a" respect to $\beta_{1}$ and $\delta_{1}$ when $\delta_{1}=11$ and $\beta_{2}=0.01$ both two cases with $v_{0}=1$. 

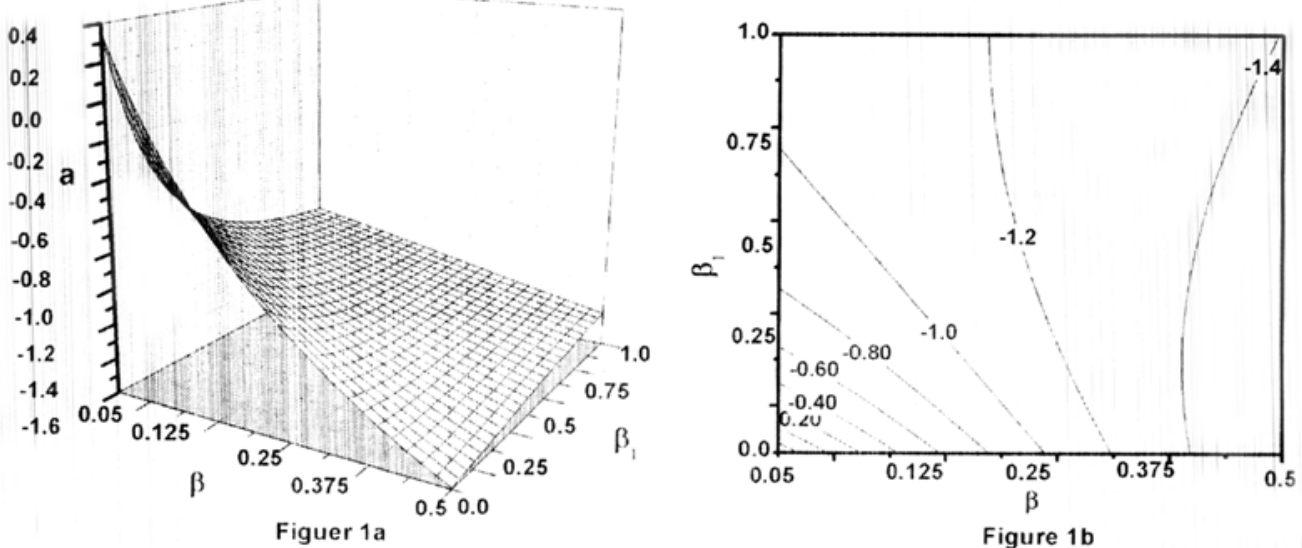

Figure 1. The parameter "a" as a function of $\beta$ and $\beta_{1}$ with $\delta_{1}=1, \delta_{2}=4$ and $v_{0}=1$. Figure $1 \mathrm{~b}$ is the contour plot of Figure 1a.

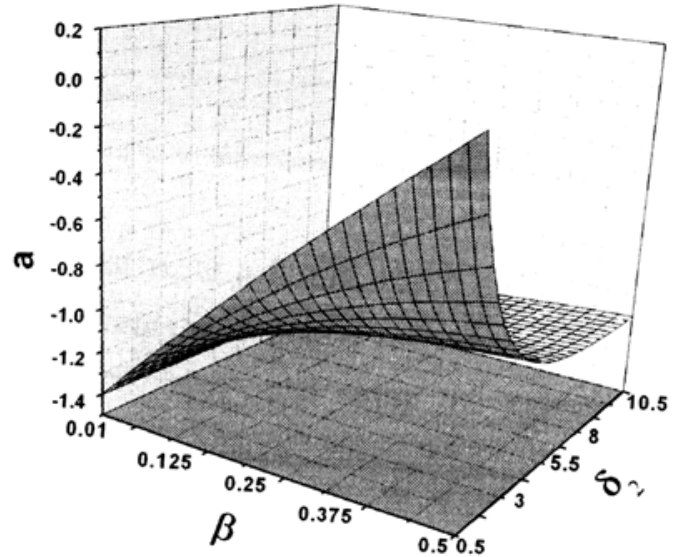

Figure 2. "a" as a function of $\beta$ and $\delta_{2}$ with $\delta_{1}=11, \beta_{1}=0.01$ and $v_{0}=1$.

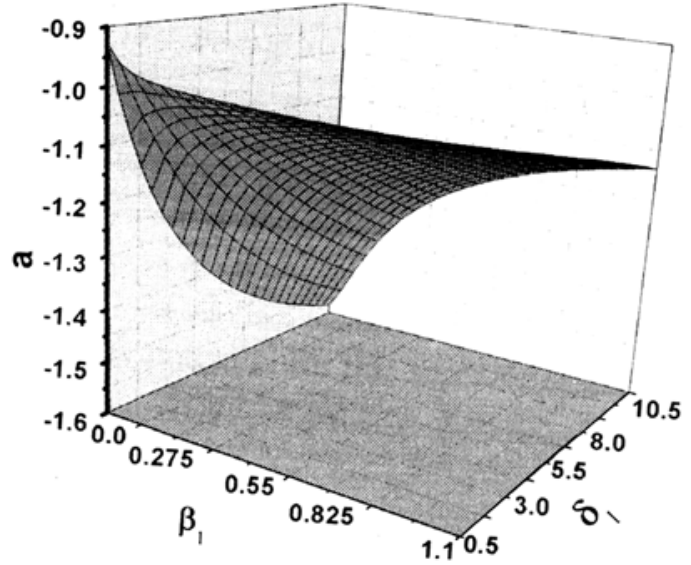

Figure 3. "a" as a function of $\beta_{1}$ and $\delta_{1}$ with $\delta_{2}=11, \beta=0.01$ and $v_{0}=1$. 
We can see that with a fixed value for $\delta_{1}$ and $\delta_{2}$, "a" reaches its maximum when $\beta$ and have their minimum values. Figure 4 presents "a" as a function of $\delta_{1}$ and $\delta_{2}$, with $\beta=0.01, \beta_{1}=0.5$ and $v_{0}=1$.
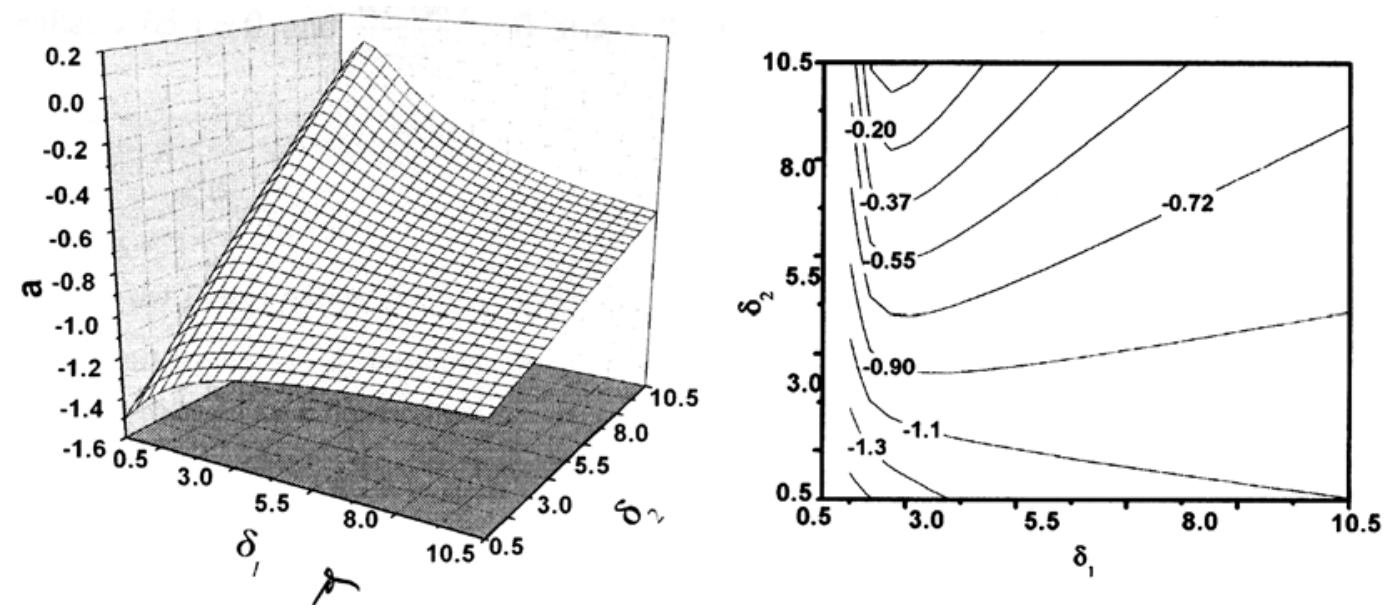

Figure 4. Parameter "a" as a function of $\delta_{1}$ and $\delta_{2}$ with $\beta=0.01, \beta_{1}=0.5$ and $v_{0}=1$.

All of the figures show that "a" is negative for most of the acceptable values of the parameters and it is positive only in small region of parameters.

\section{Discussion}

We introduce the variable

$$
\chi=l \xi+m \eta-u \tau
$$

where $\chi$ is the transformed coordinate relative to a frame which moves with the velocity $u$. "1" and " $\mathrm{m}$ " are the directional cosines of the wave vector " $\mathrm{k}$ " along the and respectively, in the way that

By integrating (29) respect to the variable iand using the vanishing boundary condition for and its derivatives up to the second-order for $|\chi| \rightarrow \infty$, we have

$$
\frac{d^{2} \phi_{1}}{d \chi^{2}}=\frac{h}{l^{4} b} \phi_{1}-\frac{a}{2 l^{2} b} \phi_{1}^{2}
$$

where

$$
h=u l-m^{2} c
$$

Equation (32) has solitonic solutions and one-soliton solution for this equation is given by

$$
\phi_{1}=\phi_{0} \sec h^{2}\left[\frac{\chi}{W}\right]
$$


where $\phi_{0}=\frac{3 h}{I^{2} a}$ is the amplitude while $W=2 \sqrt{\frac{I^{4} b}{h}}$ is the width of the soliton.

For investigating the stability conditions of this solution, we use a method based on the energy considerations [17]. Thus we are going to find the Sagdeev potential for this situation. Eq. (32) can be written as

$$
\frac{d^{2} \phi_{1}}{d \chi^{2}}=\frac{h}{l^{4} b} \phi_{1}-\frac{a}{2 l^{2} b} \phi_{1}^{2}=-\frac{d V\left(\phi_{1}\right)}{d \phi_{1}}
$$

In order to obtain the Sagdeev potential, eq. (26) is integrated to yield the nonlinear equation of motion as

where

$$
V\left(\phi_{1}\right)=\frac{a}{6 I^{2} b} \phi_{1}^{3}-\frac{h}{2 I^{4} b} \phi_{1}^{2}
$$

It is clear that $V\left(\phi_{1}\right)=0$ and $\left(d V\left(\phi_{1}\right)\right) /\left(d \phi_{1}\right)=0$ at $\phi_{1}=0$. A stable solitonic solution 2016)

II) There must exist a nonzero crossing point $\phi_{1}=\phi_{0}$ that $V\left(\phi_{1}=\phi_{0}\right)=0$.

III) There must exist a $\phi_{1}$ between $\phi_{1}=0$ and to make

Thus, from (36) and (37) we have

The parameters, $l$ and $b$ are positive. Therefore $h>0$ or

$$
u l-m^{2} c>0
$$

It is clear that the width (W) of a stable solitary wave is real.

We found that $h>0$ and also for most of the cases the parameter "a" is negative. By 
these conditions the term

is negative. Therefore the solution is a rarefactive soliton in most of the cases. Figure 5 shows the Sagdeev potential as a function of $\phi_{1}$.

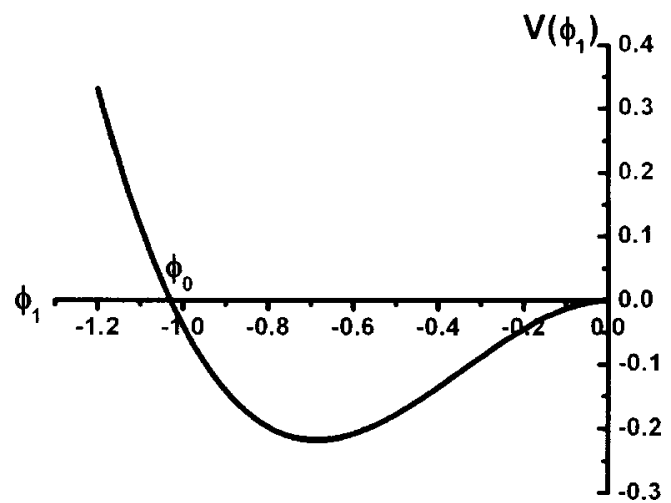

Figure 5. Sagdeev potential as a function of $\phi_{1}$.

Figure 6 presents the soliton profile for different values of $u$. All of the fucntions simulated with the values $\delta_{1}=\delta_{2}=11, \beta_{1}=0.5, \beta=0.01, v=1$ and $I=0.6$.

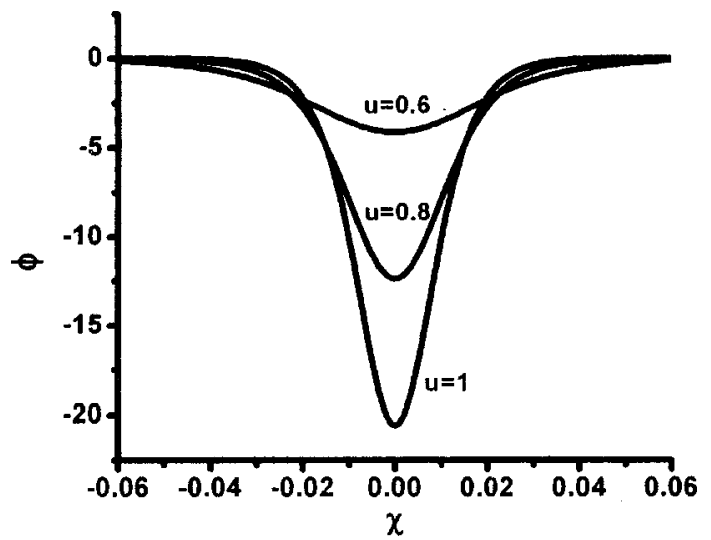

Figure 6. The shape of soliton with different values of $u$, with $\delta_{1}=\delta_{2}=11, \beta_{1}=0.5, \beta=0.01, v=1$ and $I=0.6$.

Now let us find the stability conditions for the above solution. From the (39) we have

$$
u>\frac{m^{2}}{l} c
$$

or

$$
u>\left(\frac{1-l^{2}}{l}\right) c
$$


If $\left(\left(1-I^{2}\right) / I\right)>1$ then $u>c$ and when

we have $u<c$. Thus the soliton is stable if

Figure 7 shows the soliton amplitude $\left(\phi_{0}\right)$ as a function of velocity "u" and figure 8 presents the soliton width respect to the velocity "u".

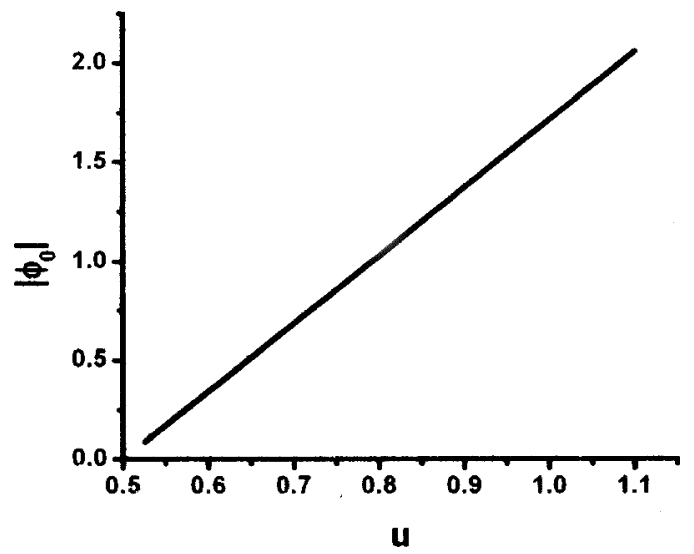

$((4 \geq \beta) /)<$ when $0<$ Figsreg. Soliton amplitude as a function of "u".

$0<u<c$ when $0.62<I<1$

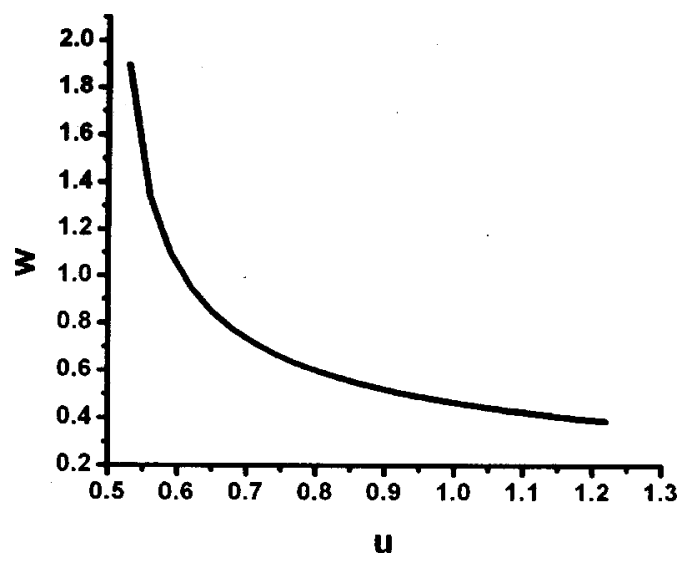

Figure 8. Soliton width as a function of velocity "u".

We can see that the amplitude of the soliton $\left(\phi_{0}\right)$ increases when "u" is increased, while its width decreases with an increasing velocity " $u$ ". On the other hand, from the definition of the soliton amplitude and its width, one can find that the amplitude (width) decreases (increases) with an increasing value for the parameter "I". This means that the parameters "u" and "l" have important roles in the stability of soliton. Thus a soliton is stable when the effects of these two phenomena cancel out each other. 
Finally for the case $m^{2} / I=1$ and $u>c$, we have

$$
W=2 \sqrt{\frac{l^{3} b}{u-c}}, \quad \phi_{0}=\frac{3(u-c)}{l a}, \phi_{1}=\phi_{0} \sec h^{2}\left(\frac{\chi}{W}\right) .
$$

And the potential is

$$
V\left(\phi_{1}\right)=\frac{a}{6 I^{2} b} \phi_{1}^{3}-\frac{(u-c)}{2 l^{3} b} \phi_{1}^{2}
$$

\section{Energy of soliton and linear dispersion relation}

The velocity components of the dust particles come from (24) and (25)

$$
\begin{aligned}
& u_{1 d}=u_{1 m} \sec h^{2}\left(\frac{\chi}{W}\right), \quad u_{1 m}=-\frac{\phi_{0}}{v_{0}} \\
& v_{1 d}=v_{1 m} \sec h^{2}\left(\frac{\chi}{W}\right), \quad u_{1 m}=-\frac{m}{1} \frac{\phi_{0}}{v_{0}} .
\end{aligned}
$$

The energy of soliton can be obtained from the following eq. [20]

$$
E_{k}=\int_{-\infty}^{\infty}\left(v_{1 d}^{2}+u_{1 d}^{2}\right) d \chi
$$

For the case $m^{2} / l=1$ we have

$$
E_{k}=\frac{\phi_{0}^{2}}{l^{2} v_{0}^{2}} \int_{-\infty}^{\infty} \sec h^{4}\left(\frac{\chi}{W}\right) d \chi=\frac{24(u-c)^{2}}{a^{2} l^{2}}\left(1+\gamma_{1}\right) \sqrt{\frac{b l^{3}}{u-c}}
$$

Figure 9 indicates that the energy of soliton increases with increasing $\gamma_{1}$.

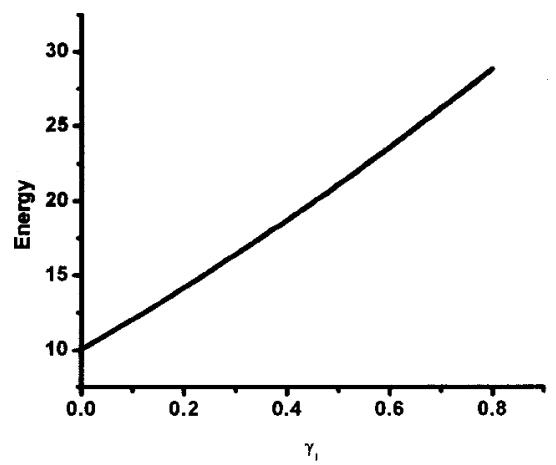

Figure 9. Energy of the soliton as a function of $\gamma_{1}$ for $\gamma_{2}=0, \delta_{1}=0.6, \delta_{2}=0.7, \beta=0.01, \beta_{1}=0.5, I=0.6$ and $u=1$. 
Figure 10 indicates that increasing $k\left(\gamma_{1}\right)$ leads to increasing (decreasing) values for the $\omega$. For real values of , all perturbation variables oscillate harmonically and if any or all of the 's have positive imaginary parts, then the system is unstable since those normal modes will grow in time [23].

\section{Conclusion and remark}

The KP equation was obtained in unmagnetized dusty plasma with variable dust charge and two temperature ions. For the KP equation (32), parameters "b" and "c" are always positive. But parameter "a" can be positive or negative ; however it is negative for most of the cases. This means that generally a rarefactive soliton is appeared in the medium. Consequently amplitude of the solitary waves is smaller as compared to the onedimensional case [16].

The Sagdeev potential was derived and stability conditions were investigated. One can find that for a stable soliton the velocity "u" has some limitations (see (4)). This means that the solitons are stable only if the effects of dust and ions motion cancel out each other. Analytically, the coefficients of the dispersive terms, "b" and "c" depend on the parameter . Indeed dispersion decreases when $\gamma_{1}$ is increased. The parameter "a" is the coefficient of the nonlinear term. It is a function of relative densities, relative temperatures, $\gamma_{1}$ and $\gamma_{2}$. Therefore, it is possible that the competition between the nonlinear term and dispersion terms, lead to the formation of a soliton. The energy of soliton and linear dispersion relation have been derived and discussed, too.

Since the parameter "a" can be positive or negative it can also be zero. But a solitonic solution can not be established when "a" is zero; therefore "a" has a critical value. This situation is very important and can be investigated in future.

\section{Acknowledgment}

We would like to thank A. R. Mokhtari for useful comments.

\section{References}

[1] P K Shukla and A A Mamum Introduction to Dusty Plasma Physics, (London, UK ; IOP) and references therein (2002)

[2] N N Rao, P K Shukla and My Yu Planet Space Sci., 38543 (1990)

[3] D A Mendis and M Rosenberg Annu Rev Astros Astrophys. 32419 (1994)

[4] C K Geortz Rev. Geophys. 27217 (1989)

[5] E C Whipple, T G Northrop and D A Mendis J. Geophys. Res. 907405 (1985)

[6] W S Duan, K P Lu and J B Zhao Chin Phys. Lett. 181088 (2001)

[7] P K Shukla, A A Mamun Introduction to Dusty Plasma (Bristol, UK : IOP Publishing) (2002)

[8] R Bharuthram and P K Shukla Planet Space Sci. 40239 (1992)

[9] Y Nakamura and A Sarma Phys. Plasmas 83921 (2001)

[10] J H He Chaos, Soliton \& Fractals 19843 (2004)

[11] T S Gill et al, Chaos, Solitons and Fractals 281106 (2006) 
[12] Y N Nejoh Phys. Plasmas 42813 (1997)

[13] W M Moslem Chaos, Solitons and Fractals 28994 (2006)

[14] F Melandso and F T Askalsen Havnes O Planet Space Sci. 41312 (1993)

[15] W S Duan Chaos, Soliton and Fractals 14503 (2002)

[16] B-S Xie, K-F He and Z-Q Huang Phys. Plasmas 63808 (1999)

[17] N Krall and A W Trivelpiece Principles of Plasma Physics, (McGraw-Hill, Inc.) (1973)

[18] W M Moslem Physics Letters A351 290 (2006)

[19] R Annou Phys. Plasmas 52813 (1998)

[20] F Verheest and S Lakhina Astrophys Space Sci 240215 (1996)

[21] N D'Angelo and R L Merlino Plant Space Sci. 441593 (1996)

[22] R Annou Phys. Plasmas 52813 (1998)

[23] N A Krall and A W Trivelpiece Principles of Physics (San Francisco, CA : San Francisco Press) (1986) 